Original Paper http://ajol.info/index.php/ijbcs http://indexmedicus.afro.who.int

\title{
Caractérisation des systèmes de production de Jatropha dans les exploitations agricoles de la zone Ouest du Burkina Faso
}

\author{
Pascal BAZONGO ${ }^{1,2^{*}}$, Karim TRAORE ${ }^{1}$, Ouola TRAORE ${ }^{1}$, Ablassé BILGO ${ }^{5}$, Barthelemy \\ YELEMOU $^{4}$, Kadidia B. SANON ${ }^{3}$, Victor HIEN ${ }^{5}$ et Bismarck H. NACRO ${ }^{2}$ \\ ${ }^{1}$ Institut de l'Environnement et de Recherches Agricoles (INERA), Département Gestion des Ressources \\ Naturelles et Système de Production, INERA-Farako-Ba, Laboratoire Sol Eau Plante, BP 910 Bobo-Dioulasso, \\ Burkina Faso. \\ ${ }^{2}$ Université Polytechnique de Bobo, Ecole Doctorale, Sciences Naturelles et Agronomie 1 BP. 1091 Bobo- \\ Dioulasso 01 Burkina Faso. \\ ${ }^{3}$ Laboratoire de Microbiologie, INERA/DPF, 03 BP 7047 Ouagadougou 03, Burkina Faso. \\ ${ }^{4}$ Institut de l'Environnement et de Recherches Agricoles (INERA), Département Gestion des Ressources \\ Naturelles et Système de Production, INERA-Saria,BP 10 Koudougou, Burkina Faso. \\ ${ }^{5}$ Institut de l'Environnement et de Recherches Agricoles (INERA), Département Gestion des Ressources \\ Naturelles et Système de Production, INERA-Kamboinsé, Faso ; Laboratoire Sol Eau Plante, 01 BP 476 \\ Ouagadougou 01, Burkina Faso. \\ ${ }^{*}$ Corresponding author, E-mail: bazpasco@yahoo.fr; 01 BP. 1091 Bobo-Dioulasso 01 Burkina Faso,
}

\section{REMERCIEMENTS}

Les auteurs traduisent leur gratitude à l'Union Européenne à travers le "10 $0^{e}$ Fond Européen de Développement". Les remerciements vont également à l'endroit du programme de subvention de recherche de l'Union Africaine [HRST/ST/AURG/CALL1/2011], pour avoir financé cette étude.

\section{RESUME}

La production agricole est la principale source de développement socio-économique au Burkina Faso. Depuis les années 2000, la promotion du Biocarburant suscite un regain d'intérêt pour la plantation de Pourghère (Jatropha curcas L.). Dans 14 villages de la zone Ouest du Burkina Faso, une enquête semistructurée basée sur un questionnaire a été appliquée auprès de 210 producteurs de Jatropha. Les résultats des enquêtes indiquent que la production de Jatropha est récente. La superficie des plantations de Jatropha varie entre 7,5 et 30 ha par village. L'écartement le plus adopté pour la plantation de Jatropha est de $5 \mathrm{~m}$ entre les lignes et $2 \mathrm{~m}$ entre les plants $(5 \mathrm{~m} \times 2 \mathrm{~m})$, soit une densité de 1071 plants/ha. Une proportion de $80,4 \%$ des producteurs enquêtés indique avoir 4 à 7 ans d'expérience dans la culture de Jatropha. Les rendements varient entre 300 et $700 \mathrm{~kg} / \mathrm{ha}$. Pour 78,5\% des exploitants, leur engagement dans la culture de Jatropha a été motivé par les structures de promotion de Jatropha. Plus de $90 \%$ des producteurs pratiquent la technique de la pépinière-transplantation et $70 \%$ pratiquent la culture associée au Jatropha. Les enquêtes révèlent que $70 \%$ des producteurs associent les légumineuses au Jatropha et que $22 \%$ des producteurs pratiquent la fertilisation de Jatropha. L'application des traitements phytosanitaires au Jatropha peu pratiquée, ne concerne que $11 \%$ des producteurs.

(C) 2015 International Formulae Group. All rights reserved.

Mots clés : Biocarburant, pourghère (Jatropha curcas L.), enquêtes, exploitants, Burkina Faso. 


\title{
Characterization of the systems of production of Jatropha in the agricultural exploitations of the West zone of Burkina Foso
}

\begin{abstract}
The agricultural production is the main source of socioeconomic development of Burkina Faso. Since the years 2000, the promotion of biofuel causes a renewal of interest toward Jatropha curcas L. In 14 villages of the West zone of Burkina Faso, a semi-structured investigation based on a questionnaire has been applied on 210 farmers of Jatropha. The results of the investigations indicate that the production of Jatropha is recent. The surfaces of plantations of Jatropha vary between 7.5 and 30 ha per village. The more adopted spacing for the plantation of Jatropha is of $5 \mathrm{~m}$ between the lines and $2 \mathrm{~m}$ between the plantations $(5 \mathrm{~m} \times 2 \mathrm{~m})$, thus a density of 1071 plants/ha. A proportion of $80.4 \%$ of producers investigated have 4 to 7 years of experience in the culture of Jatropha. The yields vary between 300 and $700 \mathrm{~kg} / \mathrm{ha}$. For $78.5 \%$ of farmers, their commitment in the production of Jatropha has been motivated by the structures of promotion of Jatropha. More than $90 \%$ of the producers practice the technique of the nursery-transplantation and $70 \%$ grow cereal associated with Jatropha. The investigations reveal that $70 \%$ of producers associate vegetables with Jatropha and that $22 \%$ of producers fertilize Jatropha fields. The rate of the application of pesticides in the field of Jatropha is little, it concerns only $11 \%$ of producers.
\end{abstract}

(C) 2015 International Formulae Group. All rights reserved.

Keywords: Biofuel, Jatropha curcas L., investigations, producers, Burkina Faso.

\section{INTRODUCTION}

$\mathrm{Au}$ Burkina Faso, la culture de Jatropha est assez récente. En effet, la production de Jatropha s'est développée au cours des 10 dernières années, grâce à plusieurs projets de développement (Traoré, 2009). Les superficies de cette culture ont été évaluées entre 70.000 et 150.000 ha et exploitées par près de 200 groupements villageois (CIRAD-2IE, 2008; Ouédraogo, 2008). Plusieurs auteurs (Pellet et al., 2007 ; Neff et al., 2008; Endelevu, 2009) rapportent que Jatropha Curcas L. constitue une piste sérieuse pour l'atténuation de l'émission des gaz à effet de serre. Un des avantages de cette plante est qu'elle s'adapte aux conditions pédoclimatiques difficiles (Ouédraogo, 2000). De nombreux travaux ont montré les effets positifs de la plante sur les propriétés des sols. C'est ainsi que Girard et al. (2011) ont conclu qu'une plante transforme plus de 5 fois sa biomasse durant une période chaude d'une journée, et près de 500 fois sa biomasse finale au cours de son cycle végétatif. Gobat et al. (2010) trouvent que la part de la biomasse aérienne des arbres qui retourne au sol est très importante. Pour d'autres auteurs comme Low et al. (2007) et Endelevu (2009), Jatropha est envahissant. En Australie occidentale, la difficulté à contrôler son expansion a conduit à interdire sa culture en 2006 (Domergue et al., 2008). La possibilité que cette culture entre en compétition avec les cultures vivrières a été évoquée par Lottman. (2008). Guillaume. (2009) soutient le caractère invasif de cette plante, en évoquant un risque d'insécurité alimentaire. Il ressort au niveau de la littérature, des conclusions parfois contradictoires concernant la culture de Jatropha. Malgré un engouement certain pour Jatropha, peu d'études ont porté sur la pratique de la culture, notamment le système de culture, de sorte que beaucoup de questions se posent aujourd'hui sur i) l'impact de sa culture sur la fertilité des sols, ii) la densité optimale de plantation, et iii) ses rendements. C'est ce qui justifie cette étude qui vise à caractériser le système de culture de Jatropha dans un contexte de changements climatiques. 


\section{MATERIEL ET METHODES}

Présentation des sites d'étude

Les enquêtes ont été conduites en milieu paysan dans la zone Sud-soudanienne du Burkina Faso, dans 14 sites répartis dans 04 Provinces (le Houet, le Kénédougou, le Tuy et la Comoé) avec un climat du type Sud soudanien. La pluviosité annuelle dans cette zone oscille entre 900 et $1200 \mathrm{~mm}$, avec une saison pluvieuse qui dure entre 4 à 5 mois. Ces sites ont été choisis en référence aux actions menées par les promoteurs de la culture de Jatropha dans ces zones et suivant un transect Sud-Ouest. La répartition des 14 sites est de 6 pour la province du Houet, 2 pour le Kénédougou, 3 pour le Tuy et 3 pour la Comoé. Les sols des sites de Farako-Bâ, Matourkou et Mê sont de types ferrugineux tropicaux lessivés (Bado, 2002). Ce sont des sols pauvres en argile, très sableux, à texture sablo-limoneuse. Sur les sites de Bama et Souroukoudougou, on rencontre des sols limoneux, sablo-limoneux, sablo-argileux, sablo-argilo-limoneux et hydromorphes (Traoré, 2012). La végétation du site est la savane arborée et la forêt claire (Guinko, 1984). La savane arborée et la forêt claire sont des types de végétation rencontrés sur les sites de Lidara et Tin (Guinko, 1984). Ces deux sites sont également parsemés d'espèces de Vittelaria paradoxa avec un sol ferralitique faiblement désaturé et remanié (CPCS, 1967) ou un lixisol (FAO, 1998). Selon le BUNASOLS (2002), on rencontre à Boni, Béréba et Gombèlèdougou des sols qui se dégradent au fil des années à cause de l'occupation et de l'exploitation anarchique des terres. Ces sols se retrouvent parmi les sols minéraux bruns, les sols peu évolués, les vertisols, les sols brunifiés, les sols à sesquioxyde de fer et de manganèse, les sols ferralitiques et les sols hydromorphes. Les unités morphologiques sont des associations comprenant des sols dominants (> 50\%), des sols associés (49-20\%) et des inclusions (< 20). A Torokoro, Madiasso et Koflandé, les sols sont surtout de type ferrugineux tropical lessivé sur les versants, avec quelques sols hydromorphes à pseudogley et des lithosols (lixisols) (Youl, 2009).

\section{Méthodologie}

Pour cette étude, un échantillon de 15 producteurs a été retenu par site, soit un échantillon de 210 producteurs au total. L'âge moyen des producteurs était de plus ou moins 43 ans. L'étude a été conduite sur 10 mois, de juin 2013 à janvier 2014.

Choix des champs et des producteurs expérimentateurs

Le critère de sélection d'un producteur au sein de l'échantillon soumis à l'enquête, est la possession d'un champ de Jatropha. Sur chaque site, quinze (15) champs ont été retenus. Le choix a été fait par les producteurs eux-mêmes à travers leur Groupement de Producteurs de Jatropha. Ce choix a tenu compte de l'écartement des pieds de Jatropha, de l'âge de la plantation et de la nature de la culture associée au Jatropha. Le Tableau 1 donne les caractéristiques des parcelles de Jatropha des producteurs expérimentateurs.

Evaluation de la perception des producteurs sur la culture du Jatropha

Une enquête semi-structurée, basée sur un questionnaire en vue de recueillir les perceptions des producteurs de la zone Ouest du Burkina Faso sur la culture de Jatropha a été conduite. Des données sur les exploitations, le niveau d'adoption de Jatropha et sur les pratiques culturales ont ainsi été collectées (Tableau 1).

\section{Analyses des données}

Les données collectées sur le site ont été analysées à l'aide du logiciel SPSS $12 \mathrm{Fr}$ et du logiciel XLSTAT version 2007 ; les moyennes des traitements ont été séparées par le test de Newan-Keuls au seuil de signification de 5\% pour vérifier l'existence de différences significatives. 


\section{RESULTATS \\ Production de Jatropha}

La superficie moyenne des plantations varie entre 0,5 et 2 ha par site (Figure 1). Les résultats des enquêtes montrent que l'expérience des producteurs dans la culture de Jatropha varie entre 2 et 8 ans. La plupart des producteurs $(80,4 \%)$ indique avoir 4 à 7 ans d'expérience dans le domaine de la production de Jatropha et $14,2 \%$ ont plus de 7 ans d'expérience (Tableau 2). Seulement 5,4\% des producteurs ont moins de 4 ans d'expérience dans la culture de Jatropha. La majorité des producteurs $(80,4 \%)$, possède des plantations en pleine production et $14,2 \%$ de producteurs ont plus de 7 ans d'expérience et constatent qu'à partir de la $7^{\mathrm{e}}$ année, la production de Jatropha se stabilise.

\section{Rendement moyens des plantations}

La Figure 2 montre que les rendements graines oscillent entre 300 à $700 \mathrm{~kg} / \mathrm{ha}$. On observe des différences hautement significatives $(\mathrm{P}<0,0001)$ entre les rendements graines comparés pour les sites. Les rendements obtenus sur le site de Béréba sont de moitié inférieurs par rapport à ceux observés sur les sites de Torokoro et Tin pour les plantations du même âge. Les meilleures productions sont obtenues dans les plantations de Torokoro et Tin d'âge supérieur à 6 ans.

\section{L'engagement des acteurs dans la production du Jatropha}

La motivation des producteurs réside surtout dans le fait que la culture de Jatropha ne demande pas d'investissement, n'entraîne pas de charge de travail élevée et peut procurer des bénéfices certains, surtout sur le plan financier. Il s'agit d'une culture de diversification qui demande moins de suivi. Certains producteurs cultivent Jatropha pour valoriser les espaces vides ou mieux rentabiliser les exploitations. Les résultats de la Figure 3 montrent que les facteurs qui ont influencé les producteurs à s'investir dans la culture de Jatropha s'expliquent pour 78,5\% des exploitants, par les actions de sensibilisation et de formation menées par des structures de promotion de Jatropha, pour $11,5 \%$ des producteurs par le biais d'un ami et pour 10 des exploitants par un membre de la famille.

\section{Mode de plantation}

Selon les producteurs et les promoteurs (APROGER et GENESE), la variété actuellement vulgarisée dans la zone Ouest du Burkina Faso est Jatropha curcas. L dont les semences proviennent du Brésil. Il existe trois (03) modes de plantation de Jatropha, à savoir, le semis direct, pratiqué par $5,7 \%$ des producteurs enquêtés, la pratique de pépinière-transplantation utilisée par $90 \%$ des producteurs, et le mode de plantation en boutures, pratiqué par $4,3 \%$ des exploitants. Les pépinières sont mises en place en saison sèche autour des habitations. Le mode plein terre est de plus en plus préféré au mode en pot grâce à de son coût réduit. Les pépinières sont mises en place entre janvier et avril. La transplantation se fait un mois avant la saison des pluies. La pépinière de pleine terre est la plus vulgarisée car elle nécessite moins d'investissement (Figure 4). Les promoteurs de Jatropha recommandent la culture du Jatropha sur des espaces dégradés afin d'éviter toute concurrence avec les autres cultures. Pour ces derniers, Jatropha est une plante rustique qui ne nécessite pas de fertilisation. Cependant, dans quatre sites de la zone d'étude (Matourkou, Gombeledougou, Torokoro, Tin), les producteurs ont observé une meilleure croissance et une meilleure productivité de Jatropha sur les sols fertiles par rapport aux sols pauvres. 


\section{Système de culture}

La densité de plantation varie d'un producteur à l'autre et cela, en fonction du système de culture mis en place, à savoir la culture pure de Jatropha, la culture de Jatropha associée à une culture vivrière ou Jatropha cultivé sous forme de haies-vives. La Figure 5 indique les systèmes de culture adoptés par les producteurs. On observe que Jatropha est cultivé en association avec les autres cultures par environ $70 \%$ des exploitants. Pour ce faire, les écartements les plus utilisés sont de $5 \mathrm{~m}$ entre les lignes et de $2 \mathrm{~m}$ entre les pieds de Jatrophas soit environ 1071 pieds par hectare. Cette technique est avantageuse parce qu'elle permet aux producteurs d'exploiter leurs terres aussi bien pour les cultures vivrières que pour la plantation de Jatropha. Les plantes de Jatropha bénéficient aussi de l'entretien apporté aux cultures vivrières. La pratique de la monoculture de Jatropha ne concerne que $10 \%$ des exploitants.

\section{Cultures associées au Jatropha}

La nature de la culture associée au Jatropha dépend de l'exploitant et varie d'un site à l'autre (Figure 6). Les légumineuses sont conseillées par $70 \%$ des producteurs comme les cultures les mieux adaptées à une association avec Jatropha du fait de leur faible exigence en fertilisants, alors que $28 \%$ des producteurs utilisent les cultures céréalières associées au Jatropha. Seulement $2 \%$ des producteurs pratiquent l'association Jatropha-coton. L'association au Jatropha d'une culture exigeante comme le coton est peu pratiquée par les producteurs, à cause de la faible qualité des sols sur lesquels est cultivé Jatropha. La décision d'associer une culture au Jatropha est généralement prise en tenant compte du faible niveau de fertilité des sols où se cultive Jatropha, mais également de la faible exigence de la culture associée en matière de fertilisation.

\section{Fertilisations et traitements phytosanitaires}

Les résultats relatifs à la fertilisation et aux traitements phytosanitaires sont présentés dans les Figures $7 \mathrm{a}$ et $7 \mathrm{~b}$. Les résultats de l'enquête montrent que $22 \%$ des producteurs apportent des fertilisants au Jatropha alors que la majorité des producteurs $(78 \%)$ ne fertilisent pas leurs champs de Jatropha (Figure 7a). L'application de fertilisants est généralement faite sur la culture associée et les plants de Jatropha peuvent de ce fait en bénéficier. La principale forme de fertilisation de Jatropha pratiquée par quelques producteurs consiste à appliquer la fumure organique aux pieds des plants de Jatropha. Le non recours aux engrais chimiques s'explique non seulement par leur coût élevé mais également par le fait que cette plante, comme le véhiculent les messages des promoteurs de Jatropha, n'aurait pas besoin de fertilisants pour se développer. Les résultats montrent que seulement $11 \%$ des producteurs pratiquent des traitements phytosanitaires dans les pépinières pour lutter contre les insectes nuisibles de Jatropha alors que $89 \%$ des producteurs ne pratiquent aucun traitement phytosanitaire (Figure 7b). Les dégâts dûs aux termites constituent un problème réel à la production. De façon générale, il ressort que Jatropha ne bénéficie pas d'une attention particulière de la part des producteurs. Les opérations d'entretien sont plutôt apportées prioritairement aux cultures vivrières associées, ce qui profite aux plants de Jatropha. 


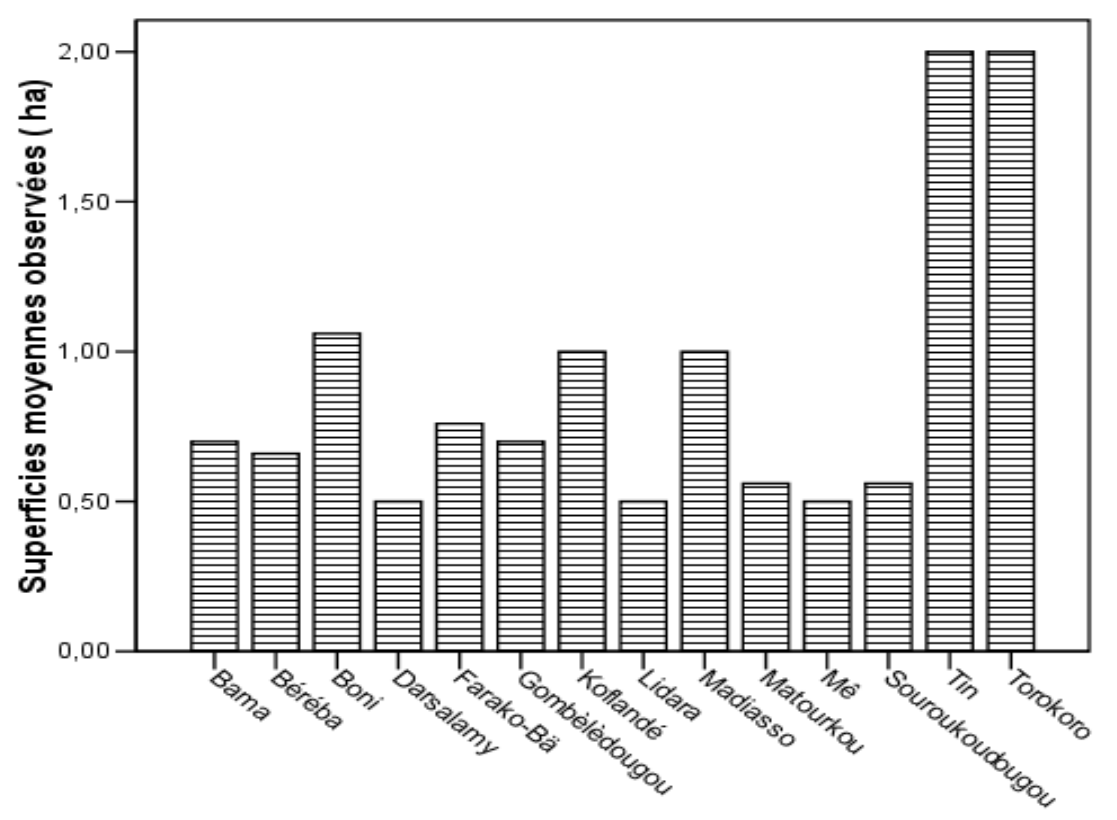

Sites de production

Figure 1 : Répartition moyenne des superficies par site.

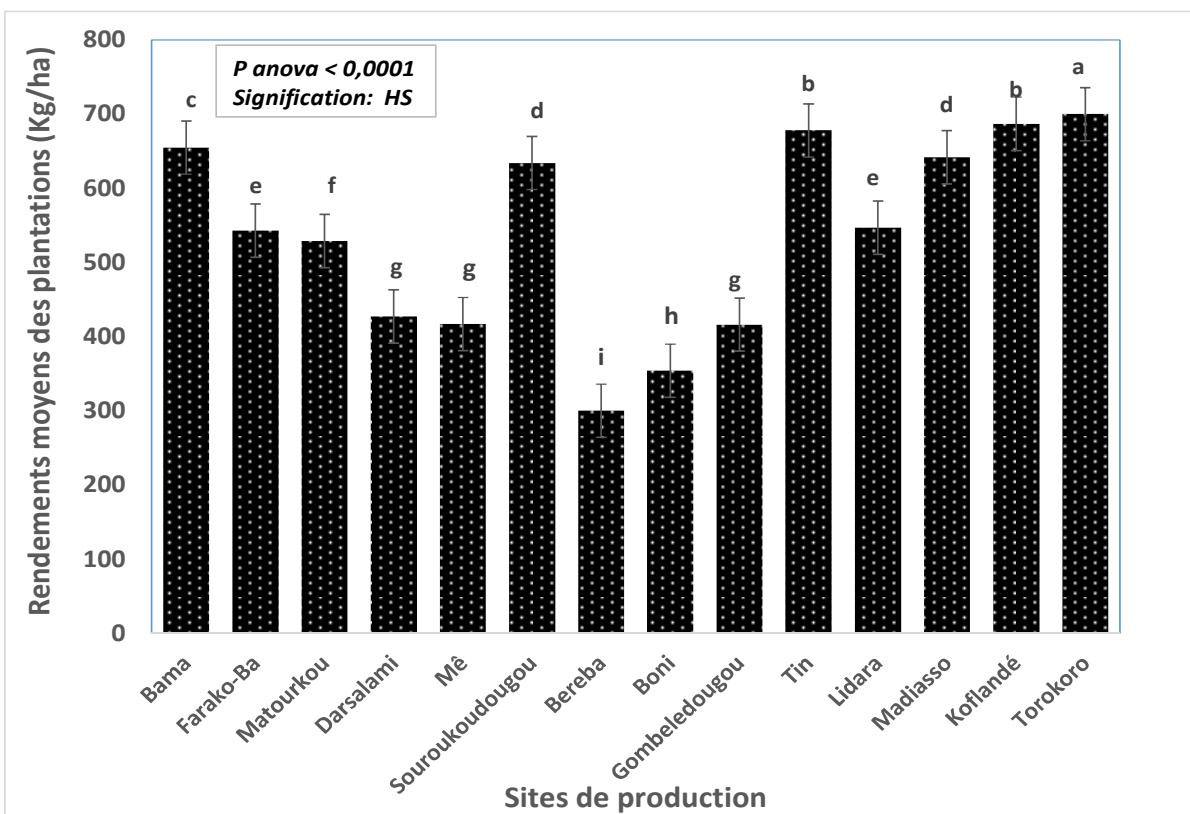

Figure 2 : Rendement moyen par site. Les moyennes affectées de la même lettre ne sont pas significativement différentes au seuil de 5\% de probabilité (test de Newman-Keuls). HS : Hautement significatif. 


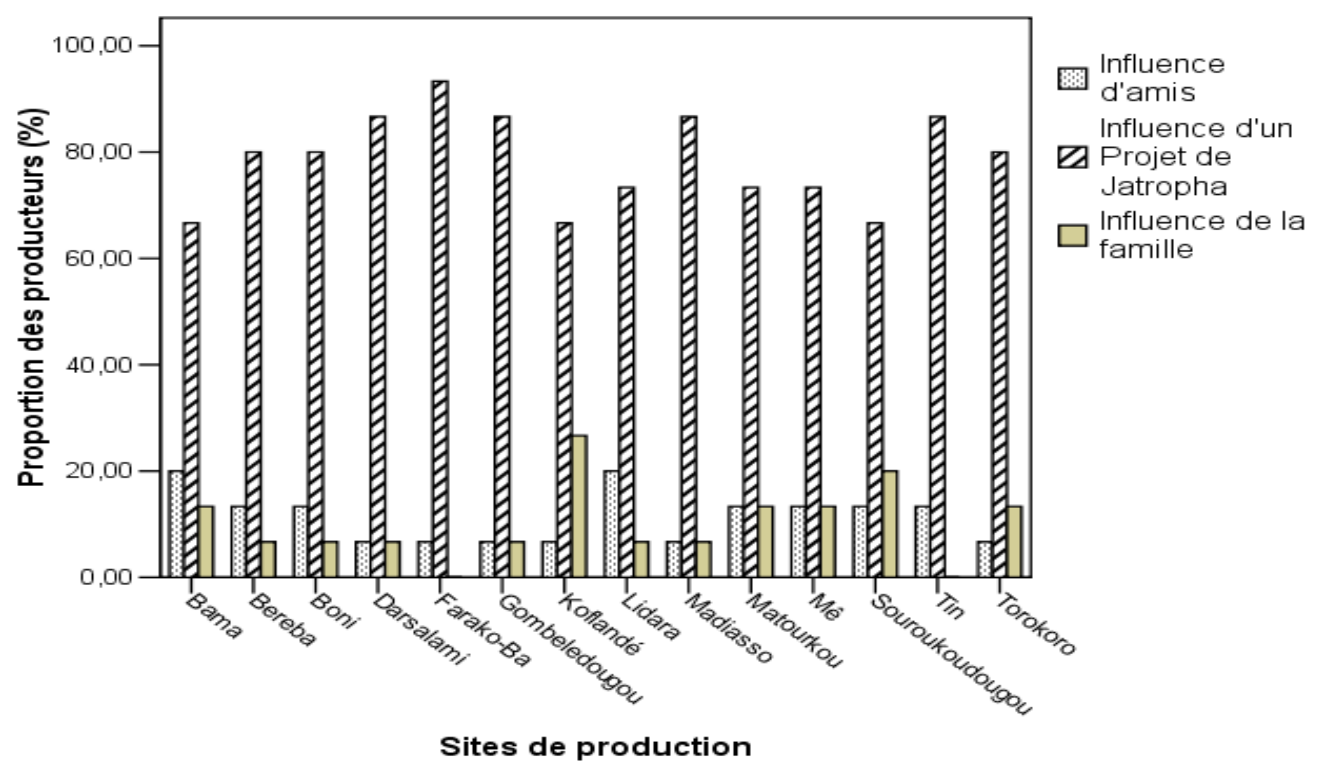

Figure 3 : Engagement des producteurs dans la production du Jatropha.

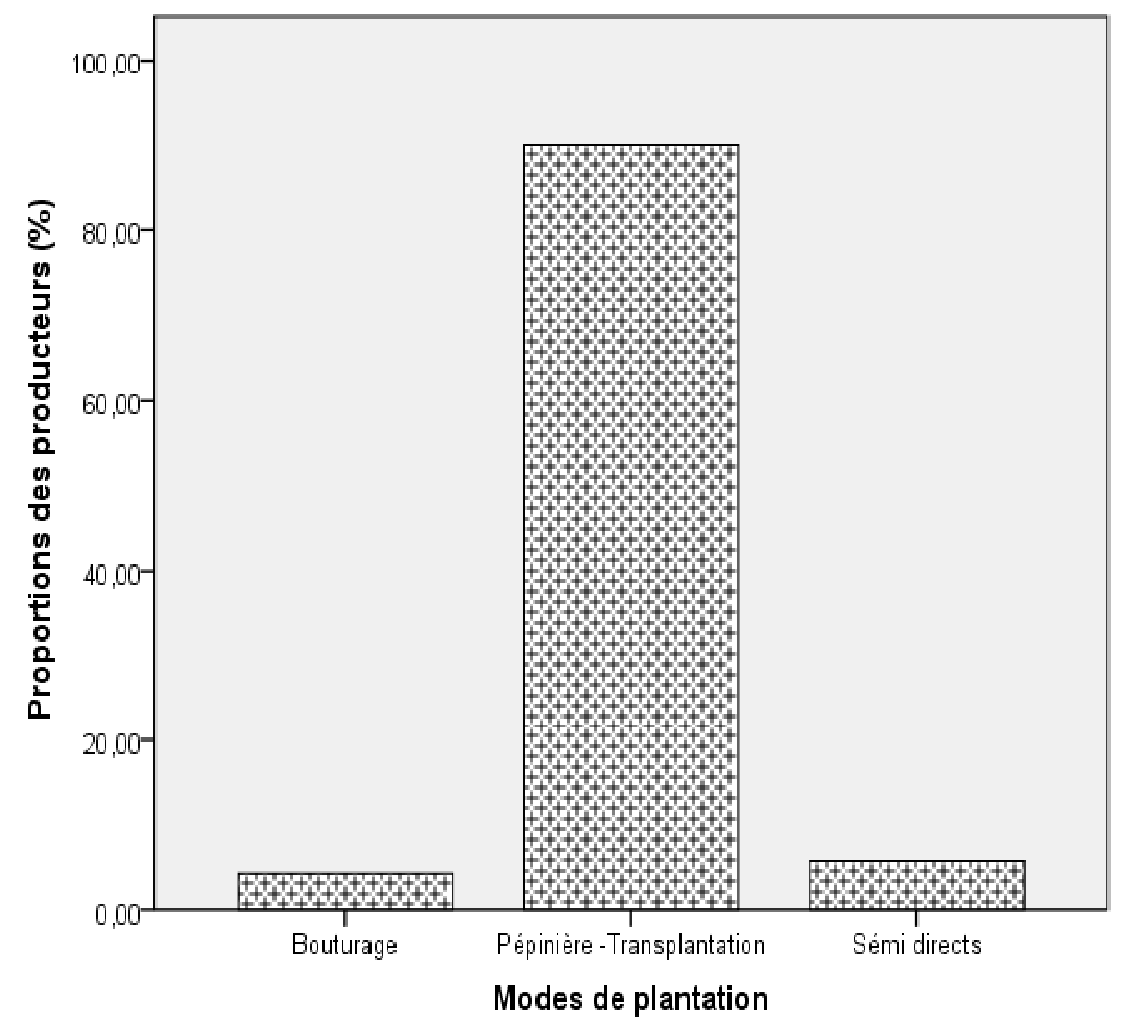

Figure 4 : Proportion des producteurs en fonction du mode de plantation. 


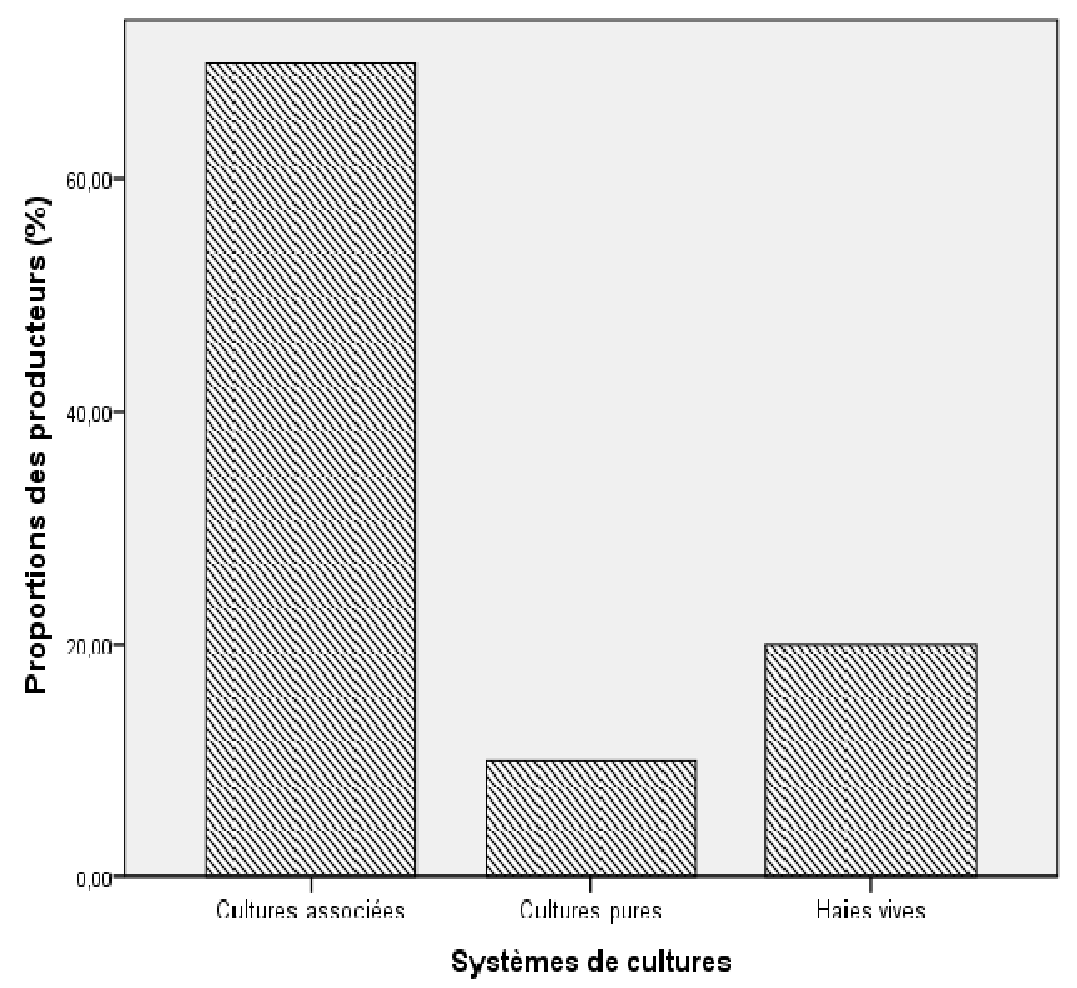

Figure 5 : Systèmes de culture à base Jatropha rencontrés.

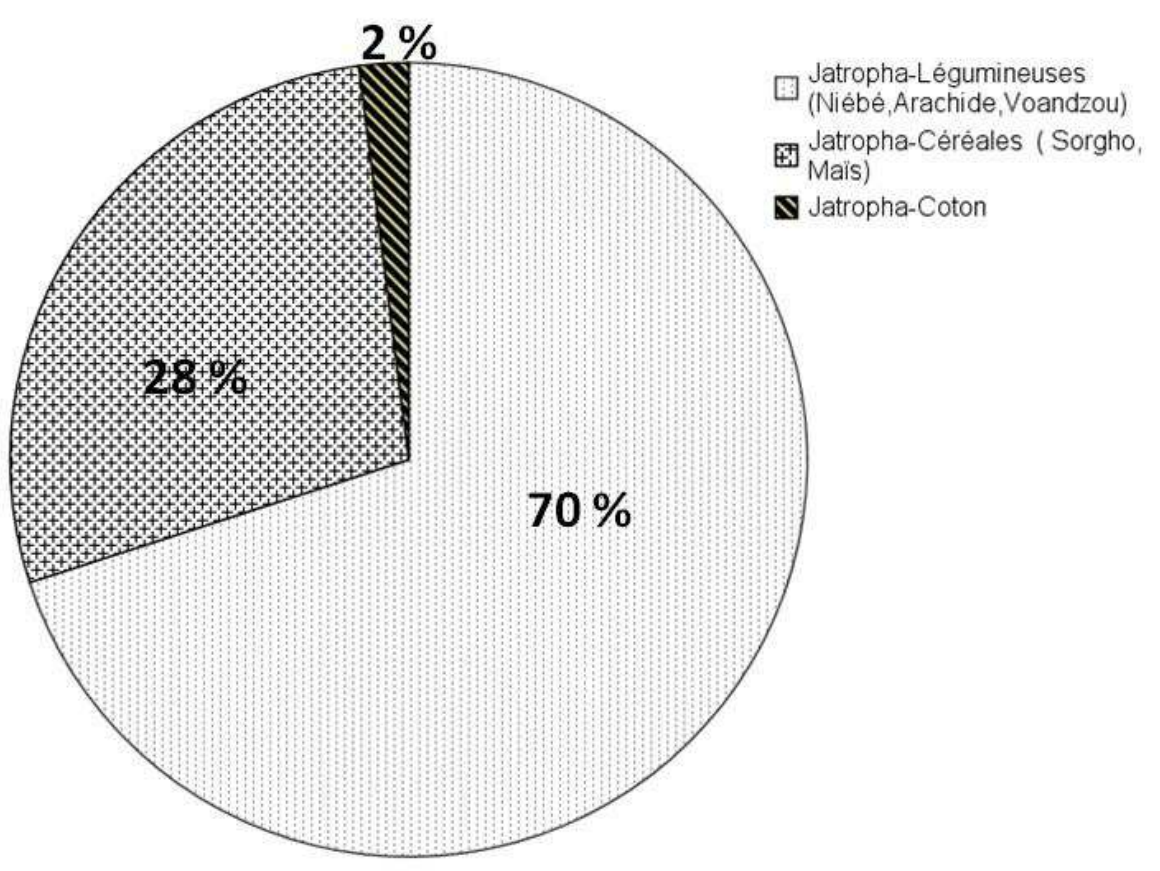

Figure 6 : Cultures associées au Jatropha. 

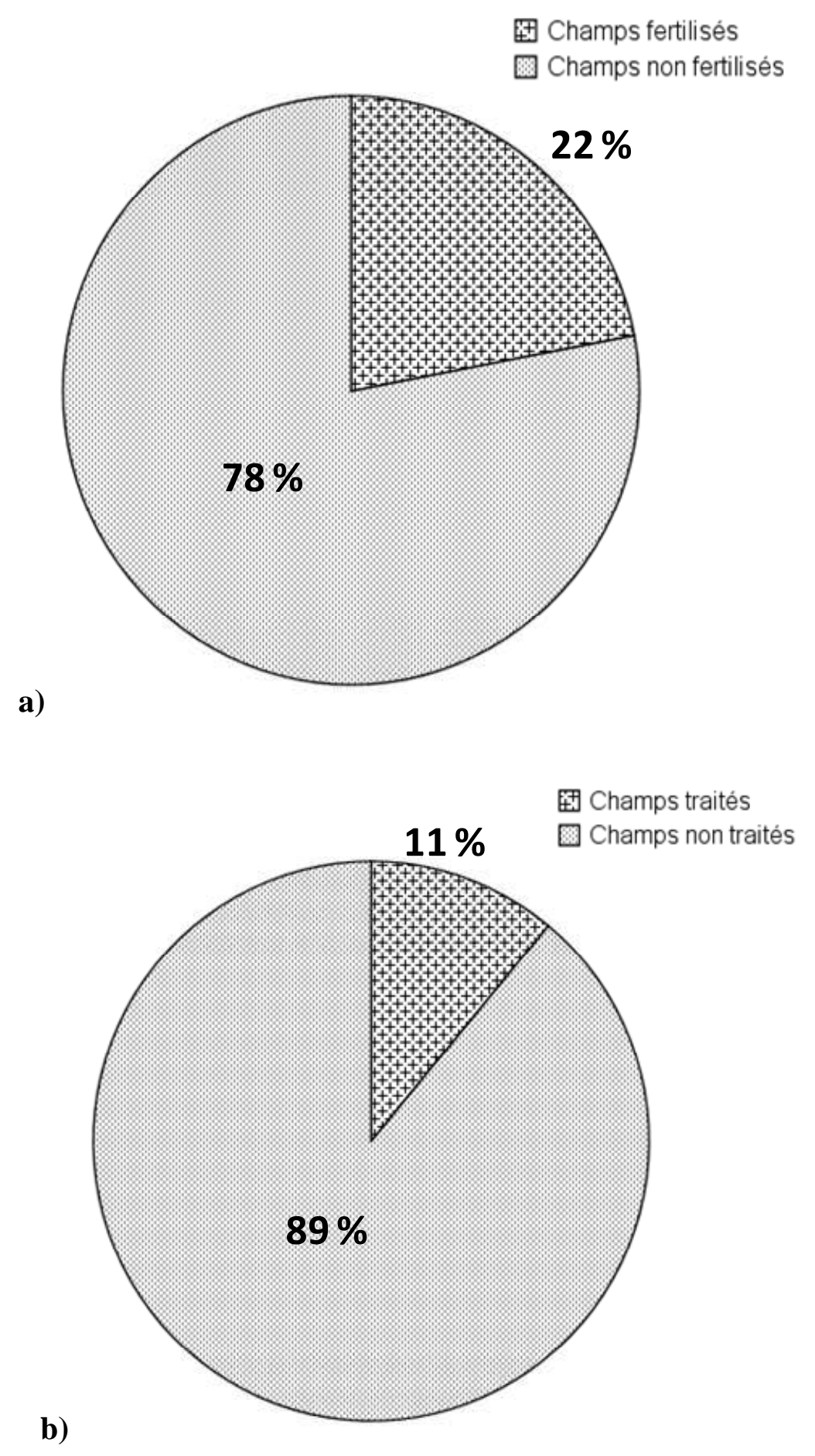

Figures 7 : Résultats de l'enquête sur a) la fertilisation et b) le traitement phytosanitaire des champs de Jatropha. 
Tableau 1 : Quelques caractéristiques des champs des producteurs expérimentateurs.

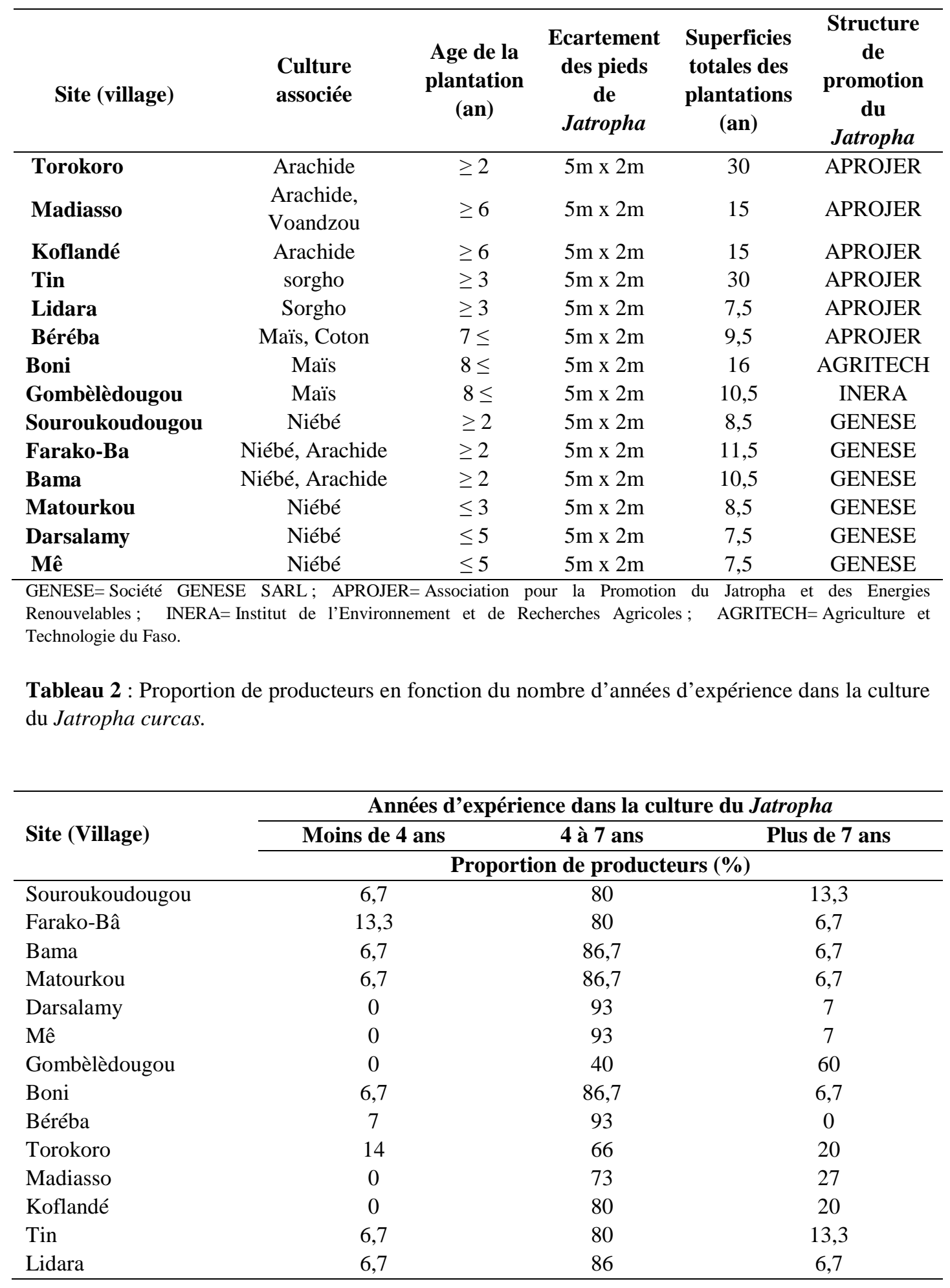




\section{DISCUSSION}

L'enquête a révélé que les superficies des plantations sont très faibles tant au niveau des producteurs, que des villages. La petite taille des exploitations s'explique, selon les producteurs, par une méfiance des producteurs liée à la non-maîtrise de la culture de Jatropha. CIRAD-2IE (2008) et Ouédraogo (2008) ont évalué entre 70.000 et 150.000 ha, la superficie totale des exploitations de Jatropha au Burkina Faso qui sont cultivées par près de 200 groupements. La culture de Jatropha est assez récente et s'est développée au cours de cette décennie (Traoré, 2009). Les enquêtes auprès des producteurs ont montré que leur engagement à produire Jatropha vient principalement de l'influence de la sensibilisation menée par des promoteurs de cette culture. Ce constat a également été fait par Traoré (2009). En effet, ces promoteurs ont axé leurs actions sur l'utilisation de la plante comme haie vive pour lutter contre l'érosion et protéger les exploitations contre les animaux en divagation (Zongo, 2007). Les producteurs n'ont observé aucune concurrence entre la culture de Jatropha et les cultures associées, ce qui confirme bien que cette culture est plutôt utilisée comme une source de diversification et ne concurrence nullement les productions vivrières. L'étude a révélé que les rendements obtenus se situent entre 300 à $700 \mathrm{~kg} / \mathrm{ha}$ selon les sites et suivant l'âge des plantations. Les rendements obtenus à travers d'autres études sur Jatropha sont très variables et fluctuent entre $250 \mathrm{~kg}$ et $5 \mathrm{t} / \mathrm{ha}$ (Pirot et al., 2012; Henning et al., 2005 ; Domergue et al., 2008). L'enquête révèle que la majorité des producteurs possède des plantations en pleine production et indique qu'à partir de la $7^{\mathrm{e}}$ année de culture, la production de Jatropha se stabilise. Les rendements de Jatropha souvent présentés dans la littérature sont issus d'essais expérimentaux sur des plantations juvéniles (1 à 3 ans). Pour Ogunwole (2007), le rendement de Jatropha ne se stabilise qu'à partir de la $7^{\text {ème }}$ année avec un potentiel de rendement de
3 à $5 \mathrm{t} / \mathrm{ha} / \mathrm{an}$ pour des écartements de $3 \mathrm{~m}$ entre les lignes à $2 \mathrm{~m}$ entre les plants. A l'issue des études, Traoré (2009) a déduit que la productivité de Jatropha dépend des conditions pédoclimatiques et de l'âge de la plantation. Janin et al. (2009) concluent que les rendements agronomiques de Jatropha en milieu sahélo-soudanien ne sont pas encore parfaitement étalonnés en raison de la grande variabilité des modes de cultures et des potentialités des sols. La plantation de Jatropha en culture associée en utilisant la technique de la pépinière par rapport à la transplantation, et les écartements de $5 \mathrm{~m}$ entre les lignes et $2 \mathrm{~m}$ entre les plants, est l'itinéraire technique adopté par les producteurs. Sanou (2010) a conclu que dans ce type de plantation, les plants sont moins denses et très bien aérées. Cependant, les plants ont tendance à occuper tout l'espace disponible à cause du nombre plus élevé de branches. En effet, le nombre de branches est important pour la production de Jatropha à inflorescence terminale ou axiale avec une production qui est proportionnelle au nombre de branches. Toutefois, les écartements peuvent varier suivant les objectifs, mais sont fortement réduits avec l'âge de la plantation. Selon Ouédraogo (2000), la réduction des écartements à $3 \mathrm{~m}$ entre les lignes et $3 \mathrm{~m}$ entre les plants ne permet plus, au-delà de la troisième année de croissance de l'arbre, la mise en place d'autres cultures, à moins d'effectuer une taille des branches. Dans le but d'une production grainière, la plantation aux écartements de $4 \mathrm{~m}$ entre les lignes et $2 \mathrm{~m}$ entre les plants, soit 1326 plants/ha se révèlent plus intéressante. Avec cet écartement, les plantes ne sont pas serrées, ne présentent aucun risque de concurrence et peuvent ainsi se ramifier et croître. Pour assurer une meilleure rentabilité de l'exploitation, les cultures vivrières sont le plus souvent associées à la plantation de Jatropha. Les enquêtes ont révélé que la majorité des producteurs pratiquent l'association Jatrophalégumineuses. Les travaux de Loss (2008) et 
PSEE (2008) ont permis de proposer aux agriculteurs, des espèces adaptées à une association avec Jatropha. Il s'agit de la pastèque, du sésame et de l'arachide. Le non recours aux engrais chimiques pour la fertilisation des plantations de Jatropha s'explique par leur coût élevé mais également par les effets indirects de la fertilisation des cultures associées sur Jatropha. Par ailleurs, les producteurs ont été informés par les promoteurs que Jatropha n'est pas exigent en fertilisants pour son développement (Traore, 2009). La quasi inexistence des traitements phytosanitaires dans la production de Jatropha s'explique par le type de culture qui y est associée. Dans notre étude, les légumineuses et les céréales utilisées comme cultures associées au Jatropha sont des cultures rustiques et adaptées aux conditions du milieu.

\section{Conclusion}

Cette étude a permis, à partir d'entretiens menés auprès des producteurs de Jatropha de faire les constats suivants: le développement de Jatropha au Burkina Faso est surtout l'œuvre des promoteurs privés ou de projets comme GENESE SARL, APROJER et AGRITECH FASO ; le mode de plantation de Jatropha le plus adopté est l'écartement de $5 \mathrm{~m}$ entre les lignes et $2 \mathrm{~m}$ entre les pieds, soit 1071 plants/ha. Les rendements observés sont inférieurs à ceux attendus. Les producteurs restent méfiants quant à associer au Jatropha des cultures exigeantes comme le maïs ou le coton ou à augmenter les superficies de Jatropha. Il apparaît nécessaire de poursuivre la caractérisation des systèmes de culture à base de Jatropha dans d'autres zones agroécologiques du pays en vue de proposer un itinéraire technique pour sa culture à l'échelle nationale.

\section{CONFLIT D'INTERET}

Les auteurs déclarent ne pas avoir de conflit d'intérêt pour cet article.

\section{CONTRIBUTION DES AUTEURS}

PB a conduit l'étude sur le terrain, a collecté et a analysé les données, et a écrit le manuscrit; KT et OT ont encadré, orienté l'étude et ont corrigé le manuscrit; BHN et $\mathrm{VH}$ ont encadré et corrigé le manuscrit; $\mathrm{AB}$, $\mathrm{BY}$ et KBS ont contribué à la correction du manuscrit.

\section{REMERCIEMENTS}

Les auteurs traduisent également leur gratitude au Projet UA Jatropha de l'Institut de l'Environnement et de Recherche Agricole (Stations de Recherche de Kamboinsé et de Farako-Bâ) pour son soutien multiforme à la réalisation de cette étude.

\section{REFERENCES}

Bado BV. 2002. Rôle des légumineuses sur la fertilité des sols ferrugineux tropicaux des zones guinéenne et soudanienne du Burkina- Faso. Thèse de Doctorat de troisième cycle, Université de Laval, Québec, p.166.

BUNASOLS. 2002. Etude morphopédologique des provinces du Houet et du Tuy, p.76.

CPCS. 1967. Classification des sols. Travaux de la Commission de Pédologie et de Cartographie des Sols (1963-1967). ENSA-Grignon, Laboratoire de PédologieGéologie, Paris, p.96.

CIRAD-2IE. 2008. Opportunités de développement des biocarburants au Burkina Faso Ministère de l'Agriculture, de l'Hydraulique et des Ressource Halieutique, p.176.

Domergue M, Pirot R. 2008. Jatropha curcas L. Rapport de synthèse bibliographique, p.111.

Endelevu E. 2009. Jatropha Reality Check: A field assessment of the agronomic and economic viability of Jatropha and other oilseed crops in Kenya, p.27.

FAO. 1998. Base de référence mondiale pour les ressources en sols. Rapport sur les 
ressources en sols du monde $n^{\circ} 84$. Rome : FAO.

Guillaume Z. 2009. Les impacts des biocarburants sur les zones humides ivoiriennes. Interview, Magazine radiophonique (Côte d'Ivoire/AMARC/ Convention Ramsar sur les zones humides/FAO), p.3.

Guinko S. 1984. Végétation de la HauteVolta; Thèse de Doctorat d'Etat, Université de Bordeaux II, France, p.318.

Girard MC, Walter C, Rémy JC, Berthelin J, Morel JI. 2011. La télédétection: méthode d'inventaire et de surveillance globale. Sol et environnement. Paris : Dunod, p. 454479. ISBN 978-2-10-054900-9 http://www.documentation.ird.fr/hor/fdi:0 10052442

Gobat JM, Arago M, Matthey W. 2010. Le Sol Vivant: Base de Pédologie-Biologie des Sols. Presse polytechnique et universitaires : Romanes ; 11-46.

Henning RK, Ramorafeno T. 2005. Le manuel Jatropha. Un guide pour l'exploitation intégrée de la plante Jatropha à Madagascar, p.20. www.habiterautrement.org/31_sud-nord/contributions31/Manuel.

Janin P, Ouédraogo F. C. 2009. Enjeux des agrocarburants au Burkina Faso : le cas du Jatropha curcas L, p.12. http://umrdeveloppement-societes.univ-paris1.fr

Loss TK. 2008. Socio-economic impact of Jatropha project on smallholder farmers in Mpanda, Tanzania. Master thesis. University of Hohenheim, p.95.

Neff C, Scheid A. 2008. Les Biocarburants : Analyse du potentiel de production de biocarburants à l'échelle internationale et en Tunisie. Etude GTZ. Université de Karlsruhe, p.29. cneffpaysages.blog. lemonde.fr/2009/08/04.

Lottmann JH. 2008. Lessons to be learned for Tunisia from the cultivation and utilization of Jatropha curcas Linné (JCL) worldwide. Word-doc.

Low T, Booth C. 2007. The Weedy Truth about Biofuels in Melbourne: the Invasive
Species Council, 1-20. DOI: 10.1007/9783-642-13440-1_9

Ogunwole JO, Patolia JS, Chaudahary D R, Gosh A, Chikara J. 2007. Improvment of the quality of a degraded Entisol with Jatropha curcas L. under an Indian SemiArid Conditions. In Expert seminar on Jatropha curcas L. Agronomy and genetics, 26-28 march 2007. Wageningen, the Netherlands: Fact Foundation.

Ouédraogo AD. 2008. Culture de Jatropha Une réalité à Bantogdo. Burkina Faso, p.4.

Ouédraogo M. 2000. Etude biologique et physiologique du pourghère, Jatropha curcas L. Université de Ouagadougou, Burkina Faso. Thèse d'état, p.290.

Paramathma M, Venkatachalam P, Sampathrajan A, Balakrishnan A, Jude Sudhakar R, Parthiban KT, Subramanian P, Kulanthaisamy S. 2007. Cultivation of Jatropha and Biodiesel Production. Professor and Nodal Officer, Center of Excellence in Biofuels. Agricultural Engineering College \& Research Institute, Tamil Nadu Agricultural University, Coimbatore, 817-828. DOI: http://dx.doi. org/10.1016/j.indcrop.2015.07.028

Pellet JD, Pellet E. 2007. Jatropha, le meilleur des biocarburants : mode d'emploi, histoire et avenir d'une plante extraordinaire. Lausanne (Favre), p.1.

Pirot R, Hamel O. 2012. Les réalités du Jatropha confrontés aux opportunités des mécanismes financiers liés au carbone, $\mathrm{p}$. 32.

PSEE. 2008. Situation des plantations de Jatropha au Sénégal (hivernage 2008). Note agronomique $\mathrm{n}^{\circ} 2$ déc, 2008, 10-11.

Sanou F. 2010. Productivité du Jatropha curcas L et impact de la plante sur les propriétés chimiques du sol: cas de Bagré (Centre Est du Burkina Faso). Mémoire de fin d'étude, IDR/Université Polytechnique de Bobo Dioulasso, p.55.

Traore F. 2012. Optimisation de l'utilisation des ressources en eau du Bassin du Kou pour des usages agricoles. Thèse de 
Doctorat en Science/ Université de Liège, p.163.

Traore K. 2009. Synthèse Bibliographique sur le Jatropha curcas à l'Ouest du Burkina. INERA. GRN/SP. Rapport d'activités. Burkina Faso, p.8.

Wijaya Andi, Susantidiana. 2009. "Flower characteristics and the yield of Jatropha (Jatropha curcas L.) accessions." HAYATI Journal of Biosciences 16(4): 123-126. DOI : http://dx.doi.org/10.4308/hjb.2009. 16.4.123
Youl S. 2009. Dynamique et modélisation de la dynamique du carbone dans un agrosystème de savane de l'ouest du Burkina Faso. Thèse de Doctorat Unique, UPB (Burkina Faso), p.186.

Zongo JD. 2007. Etude de la reprise des boutures en fonction de la profondeur de plantation de la longueur chez Jatropha curcas. Mémoire de fin d'études. Université polytechnique de BoboDioulasso, Institut du Développement Rural, Burkina Faso, p.34. 